\title{
NEW PHENOLOGICAL ANOMALIES REGARDING THE FLOWERING OF SPONTANEOUS AND CULTIVATED PLANTS FROM DIFFERENT PARTS OF ROMANIA
}

\author{
Aurelian Leonardo Ilie ${ }^{1, *}$, Mariana Marinescu ${ }^{2}$, Ilie Hoza ${ }^{3}$ \\ 1 "Nicolae Jiga" Theoretical Highschool, Republicii Street, No. 36, Tinca, Romania \\ ${ }^{2}$ Teacher Training Department, University of Oradea, Universității Street, No. 1, Oradea, Romania \\ 3"I Iustin Iliesiu" Gymnasium School, Anies, Romania
}

\begin{abstract}
The paper presents new observations about the phenological anomalies regarding the flowering of spontaneous and cultivated plants from different parts of Romania during the period 2010-2019.The consequences of global warming, these anomalies have become more proeminent particularly in the last eight years (2013-2019) being observed in 36 species of spontaneous and cultivated plants. Flowering extension were observed, sometimes even supplementary flowerings, fecundation and even fructification, in the cold season (November-December) and are due to the positive temperatures from the cold season and the high temperatures from spring. Most phenological anomalies were observed in the following botanical families: Rosaceae (7 species, 19.44\%), Lamiaceae (5 species, 13.88\%), Asteraceae (4 species, $11.11 \%$ ) and the least anomalies were observed in botanical families (Corylaceae, Papaveraceae, Caprifoliaceae, Hippocastanaceae, Aristolochiaceae, Brassicaceae, Geraniaceae, Primulaceae, Caryophylaceae, Solanaceae, Vitaceae, Cannaceae, Magnoliaceae with 1 species $2.77 \%$ ).
\end{abstract}

Keywords: cultivated plants, flowering, phenological anomalies, Romania, spontaneous.

\section{INTRODUCTION}

The climatic changes in the structure of seasons were determined by the global warning: premature and short springs, dry and sultry summers, autumns with high temperatures, mild winters, the substitution of the snows with rains.

Prematurely flowering, flowering extension, the existence of some supplementary flowerings, fecundation and fructification, in the cold season (November - December) are only some phenological aspects observed in plants, as a consequence of global warming effects. Generally, worldwide, phenological research on plants has focused on a single species or small group of species usually cultivated or forested. Studies on the phenology of a group or extended groups of plant species, especially at present, in the context of new climate change, are very few ( Menzel, 2003; Wang et al., 2015).

This paper is a synthesis of the observations performed by the authors in different parts of Romania regarding the influence of global warming on the flowering of spontaneous and cultivated plants.

Data about the phenological anomalies regarding the flowering of spontaneous plants in different parts of Romania were published by some authors (Ilie et al., 2018). 


\section{Current Trends in Natural Sciences}

Vol. 9, Issue 17, pp. 194-200, 2020

https://doi.org/10.47068/ctns.2020.v9i17.023

Current Trends in Natural Sciences (on-line)

ISSN: 2284-953X

Current Trends in Natural Sciences (CD-Rom)

ISSN: 2284-9521

ISSN-L: 2284-9521

ISSN-L: 2284-9521

\section{MATERIALS AND METHODS}

The observations were made during the period 2010-2019, in some counties, with the altitude ranging from 130 to $500 \mathrm{~m}$, as follows: Tinca, Râpa, Oradea, Salonta, Miersig (Bihor county, the north-wester part of Romania ), hilly areas; Maieru-Anieş (Bistrița-Năsăud county, the northern part of Romania), hilly area; Lancrăm (Alba county, central part of Romania), Căciulata, RâmnicuVâlcea (Vâlcea county, central part of Romania), hilly areas; Belinț (Timiş county, western part of Romania), plain area; Strehaia, Batoți, Drobeta Turnu-Severin (Mehedinți county), Craiova (Dolj county), Piscani, Mărăcineni (Argeş county) hilly areas. Research on phenological abnormalities in plants was performed spontaneously, not having a predetermined purpose in this regard, being carried out on the occasion of different excursions in different locations in Romania.

The identification of plant species was made using different books (Săvulescu, 1952-1976; Todor, 1968; Ciocârlan, 2000; Sârbu et al., 2013).

\section{RESULTS AND DISCUSSIONS}

During analyzed period, 2010-2019, phenological anomalies were observed regarding flowering in the 36 species of spontaneous and cultivated plants (table 1 ).

Table 1. Phenological anomalies regarding the flowering of spontaneous and cultivated plants from two parts of Romania

\begin{tabular}{|c|c|c|c|c|}
\hline Name of the species & $\begin{array}{l}\text { Data of observations in } \\
\text { different areas } \\
\text { (see legend) }\end{array}$ & $\begin{array}{l}\text { Temperature } \\
\text { (Celsius } \\
\text { degrees) }\end{array}$ & $\begin{array}{l}\text { Phenological } \\
\text { anomalies of } \\
\text { flowering }\end{array}$ & $\begin{array}{l}\text { Period normal } \\
\text { flowering, } \\
\text { months }\end{array}$ \\
\hline $\begin{array}{l}\text { Chrysanthenum morifolium Ramat, } \\
1763\end{array}$ & $1-5$ XII 2018 A & $6-7^{0} \mathrm{C}$ & E.f. & IX-XI \\
\hline Nepeta cataria Linnaeus, 1758 & 15-20 XI $2018 \mathrm{~A}$ & $8-16^{0} \mathrm{C}$ & S.f. & $\mathrm{V}-\mathrm{VII}$ \\
\hline Rosa chinensis Jacquard, 1800 & $\begin{array}{l}10 \text { XI } 2018 \text { A } \\
1-29 \text { XI } 2018 \text { T }\end{array}$ & $\begin{array}{l}5-18^{0} \mathrm{C} \\
7-20^{0} \mathrm{C}\end{array}$ & E.f. & $\mathrm{V}-\mathrm{X}$ \\
\hline Melissa officinalis Linnaeus, 1758 & $25 \mathrm{X}-1 \mathrm{XI} 2018 \mathrm{~A}$ & $18-23{ }^{0} \mathrm{C}$ & S.f. & $\mathrm{VI}-\mathrm{VIII}$ \\
\hline Calendula officinalis Linnaeus, 1758 & $25 \mathrm{X}-1 \mathrm{XI} 2018 \mathrm{~A}$ & $18-23{ }^{0} \mathrm{C}$ & E. f. & VI- XI \\
\hline Taraxacum officinale Weber, 1863 & $25 \mathrm{X}-3 \mathrm{XI} 2018 \mathrm{~A}$ & $17-23^{0} \mathrm{C}$ & S.f. & $\mathrm{IV}-\mathrm{VI}$ \\
\hline Salvia officinalis Linnaeus, 1758 & 26 XI - 2 XII 2018 A & $17-23^{0} \mathrm{C}$ & S.f. & V-VII \\
\hline Corylus avellana Linnaeus, 1758 & $\begin{array}{ll}15 \mathrm{X}-10 \text { XII } 2018 & \mathrm{~T} \\
20 \mathrm{XI}-6 \text { XII } 2018 & \mathrm{~A}\end{array}$ & $\begin{array}{l}4-25^{0} \mathrm{C} \\
5-15^{0} \mathrm{C}\end{array}$ & S.f. & II - IV \\
\hline Betula pendula Roth, 1828 & $\begin{array}{l}22 \text { XI }-15 \text { XII } 2018 \text { A } \\
23 \text { X }-19 \text { XII } 2018 \text { T }\end{array}$ & $\begin{array}{l}4-23^{0} \mathrm{C} \\
3-25^{0} \mathrm{C}\end{array}$ & S.f. & IV- V \\
\hline Symphytum officinale Linnaeus, 1758 & 15 XI - 1 XII 2018 A & $5-17^{0} \mathrm{C}$ & S.f. & $\mathrm{V}-\mathrm{VIII}$ \\
\hline Trifolium pratense Linnaeus, 1758 & $2 \mathrm{XI}-4$ XII $2018 \mathrm{~A}$ & $3-20^{\circ} \mathrm{C}$ & S.f. & $\mathrm{V}-\mathrm{IX}$ \\
\hline Melilotus officinalis Lamarck, 1753 & $5 \mathrm{XI}-27 \mathrm{XI} 2018$ A & $5-18^{0} \mathrm{C}$ & S.f. & $\mathrm{VI}-\mathrm{IX}$ \\
\hline Alnus incana Moench, 1786 & $30 \mathrm{X}-29 \mathrm{XI} 2018 \mathrm{~A}$ & $4-20^{0} \mathrm{C}$ & S.f. & III - IV \\
\hline Robinia pseudoacacia Linnaeus, 1753 & $\begin{array}{l}10-15 \text { VIII } 2018 \mathrm{~T} \\
24 \text { VII - 5 VIII 2018Ca } \\
15-18 \text { VIII } 2018 \text { La } \\
29 \text { VI-25VII 2019 A, } \\
\text { Bihor, Mehedinți counties } \\
\text { 7-12 VII 2019 Pi } \\
\text { 17-23VII 2019 Mă } \\
\text { 22-27 IX Mi }\end{array}$ & $\begin{array}{l}26-30^{0} \mathrm{C} \\
25-33^{0} \mathrm{C} \\
24-30^{0} \mathrm{C} \\
25-37^{0} \mathrm{C} \\
\\
25-27^{0} \mathrm{C} \\
24-27^{0} \mathrm{C} \\
22-25^{0} \mathrm{C} \\
\end{array}$ & $\begin{array}{l}\text { S.f.(second } \\
\text { flowering) } \\
\text { S.f. } \\
\text { S.f. } \\
\text { S.f. } \\
\text { S.f.(third } \\
\text { flowering) }\end{array}$ & $\mathrm{V}-\mathrm{VI}$ \\
\hline Papaver rhoeas Linnaeus, 1758 & $16-20$ VIII $2018 \mathrm{~T}$ & $27-33^{0} \mathrm{C}$ & S.f. & $\mathrm{V}-\mathrm{VII}$ \\
\hline Sambucus nigra Linnaeus, 1758 & $15-20$ VIII $2018 \mathrm{La}$ & $28-30^{\circ} \mathrm{C}$ & S.f. & $\mathrm{V}-\mathrm{VII}$ \\
\hline
\end{tabular}




\section{Current Trends in Natural Sciences}

Vol. 9, Issue 17, pp. 194-200, 2020

https://doi.org/10.47068/ctns.2020.v9i17.023

Current Trends in Natural Sciences (on-line)

ISSN: 2284-953X

Current Trends in Natural Sciences (CD-Rom)

ISSN: 2284-9521

ISSN-L: 2284-9521

ISSN-L: 2284-9521

\begin{tabular}{|c|c|c|c|c|}
\hline $\begin{array}{l}\text { Aesculus hipocastanum Linnaeus, } \\
1758\end{array}$ & 24 VIII - 10 IX $2018 \quad$ O & $23-28^{0} \mathrm{C}$ & S.f. & $\mathrm{V}-\mathrm{VII}$ \\
\hline $\begin{array}{l}\text { Aristolochia clematitis Linnaeus, } \\
1758\end{array}$ & $27 \mathrm{VIII}-7$ IX $2018 \quad \mathrm{O}$ & $20-27^{0} \mathrm{C}$ & S.f. & $\mathrm{V}-\mathrm{VI}$ \\
\hline Brassica rapa Linnaeus, 1758 & 19 IX $2018 \quad \mathrm{Be}$ & $26^{0} \mathrm{C}$ & E.f. & IV - VIII \\
\hline Prunella vulgaris Linnaeus, 1758 & $20 \mathrm{X}-17 \mathrm{XI} 2018 \mathrm{~T}$ & $10-21^{0} \mathrm{C}$ & S.f. & VI - VIII \\
\hline Hedera helix Linnaeus, 1758 & $10 \mathrm{VIII} 2018 \mathrm{~T}$ & $34^{0} \mathrm{C}$ & P.f. & IX - X \\
\hline Salvia nemorosa Linnaeus, 1758 & $20 \mathrm{X}-28 \mathrm{XI} 2018 \mathrm{~T}$ & $4-25^{0} \mathrm{C}$ & S.f. & VI - VIII \\
\hline Anagallis arvensis Linnaeus, 1758 & $20-26$ XI 2018 T & $4-10^{0} \mathrm{C}$ & S.f. & $\mathrm{VI}-\mathrm{IX}$ \\
\hline Gypsophila muralis Linnaeus, 1758 & $19 \mathrm{X}-27 \mathrm{XI} 2018 \mathrm{~T}$ & $4-23^{0} \mathrm{C}$ & S.f. & $\mathrm{VI}-\mathrm{IX}$ \\
\hline Anchusa officinalis Linnaeus, 1758 & $14 \mathrm{X}-23 \mathrm{XI} 2018 \mathrm{~T}$ & $5-21^{0} \mathrm{C}$ & S.f. & $\mathrm{V}-\mathrm{VII}$ \\
\hline Nicotiana alata Link et Otto, 1876 & $26 \mathrm{X}-21 \mathrm{XI} 2018 \mathrm{~T}$ & $5-23^{0} \mathrm{C}$ & E.f. & VII $-\mathrm{X}$ \\
\hline Vitis vinifera Linnaeus, 1753 & 20 IX -3 XI $2018 \quad$ T & $23-25^{\circ} \mathrm{C}$ & $\begin{array}{l}\text { S.f. } \\
\text { Fe } \\
\text { Fr }\end{array}$ & $\mathrm{V}-\mathrm{VI}$ \\
\hline Canna indica Linnaeus, 1753 & 5 VIII - 20 IX $2018 \mathrm{~T}$ & $21-30^{\circ} \mathrm{C}$ & E.f. & VIII - IX \\
\hline Rosa canina Linnaeus, 1753 & $\begin{array}{l}22 \text { IX } 2018 \mathrm{Sa} \\
31 \times 2015 \quad \mathrm{~T}\end{array}$ & $\begin{array}{l}23^{0} \mathrm{C} \\
15^{0} \mathrm{C}\end{array}$ & S.f. & VI - VII \\
\hline Prunus cerasifera Linnaeus, 1753 & $\begin{array}{l}19 \text { IX } 2018 \mathrm{St} \\
20 \mathrm{IX}-27 \text { X } 2018 \mathrm{~T} \\
18-28 \text { IX } 2018 \text { Dolj, } \\
\text { Vâlcea counties }\end{array}$ & $\begin{array}{l}27^{0} \mathrm{C} \\
25-27^{0} \mathrm{C} \\
25-28^{0} \mathrm{C}\end{array}$ & S.f. & $\mathrm{IV}-\mathrm{V}$ \\
\hline Cerasus avium Moench, 1786 & $\begin{array}{l}19 \mathrm{IX} 2018 \mathrm{St} \\
17-30 \mathrm{IX} 2018 \mathrm{Ra} \\
18 \mathrm{IX} 2018 \mathrm{D} \\
21 \mathrm{IX} 2018 \mathrm{Rm} \mathrm{V}\end{array}$ & $\begin{array}{l}27^{0} \mathrm{C} \\
25-27^{0} \mathrm{C} \\
24^{0} \mathrm{C} \\
26^{0} \mathrm{C}\end{array}$ & S.f. & $\mathrm{IV}-\mathrm{V}$ \\
\hline Prunus domestica Linnaeus, 1753 & $\begin{array}{l}20 \text { IX }-6 \text { X } 2018 \mathrm{~T} \\
18-28 \mathrm{IX} 2018 \text { Dolj }\end{array}$ & $24-26^{0} \mathrm{C}$ & S.f. & IV - V \\
\hline Malus pumila Miller, 1768 & $\begin{array}{l}17-30 \text { IX } 2018 \mathrm{Ra} \\
17-30 \text { IX } 2018 \mathrm{~T} \\
18-28 \text { IX } 2018 \text { Dolj }\end{array}$ & $\begin{array}{l}25-27^{0} \mathrm{C} \\
25-27^{0} \mathrm{C} \\
24-27^{0} \mathrm{c}\end{array}$ & S.f. & $\mathrm{IV}-\mathrm{V}$ \\
\hline Magnolia denudata Desroussses, 1792 & $\begin{array}{l}23 \mathrm{VI}-12 \mathrm{VII} \mathrm{Cv} \\
30 \mathrm{VI}-158 \text { VII O } \\
\end{array}$ & $26-34^{0} \mathrm{C}$ & S.f. & III \\
\hline Crataegus monogyna Linnaeus, 1753 & $28-31 \times 2010 \mathrm{~T}$ & $14-15^{0} \mathrm{C}$ & S.f. & $\mathrm{V}-\mathrm{VI}$ \\
\hline
\end{tabular}

Legend: A - Anieş (BN), T - Tinca (BH), Ca - Căciulata; Rm-Râmnicu -Vâlcea (VL), La - Lancrăm (AB), O - Oradea (BH), Be - Belinț (TM), Ra - Râpa (BH), Sa - Salonta (BH), St - Strehaia (MH), D - Dârmăneşti (AG), Mi-Miersig (BH), Cv - Craiova (DJ), Pi - Piscani, Mă - Mărăcineni (AG), E.f. - extension flowering, S.f. - supplementary flowering, Fe - fecundation, Fr - fructification, P.f. - prematurely flowering.

I,II,X.....XII-months of the year (January - December)

Temperatures were measured directly in nature, the data did not come from weather stations. The observed species belong to 18 families.

Three families are best represented: Rosaceae ( 7 species, 19.44\%), Lamiaceae (5 species, 13.88\%), and Asteraceae (4 species, 11.11\%) being followed by Fabaceae (3 species, 8.33\%), Betulaceae (2 species, $5.55 \%$ ), Boraginaceae ( 2 species, $5.55 \%$ ), Corylaceae (1 species, $2.77 \%)$, Papaveraceae (1 species, 2.77\%), Caprifoliaceae (1 species, 2.77\%), Hippocastanaceae (1 species, 2.77\%), Aristolochiaceae (1 species, 2.77\%), Brassicaceae (1 species, 2.77\%), Araliaceae (1 species, 2.77\%), Primulaceae (1 species, 2.77\%), Caryophylaceae(1 species, 2.77\%), Solanaceae (1 species, 2.77\%), Vitaceae (1 species, 2.77\%), Cannaceae (1 species, 2.77\%), Magnoliaceae (1 species, $2.77 \%$ ). There were observed prematurely flowering in one species of plant, supplementary 


\section{Current Trends in Natural Sciences}

Vol. 9, Issue 17, pp. 194-200, 2020

https://doi.org/10.47068/ctns.2020.v9i17.023

Current Trends in Natural Sciences (on-line)

ISSN: 2284-953X

Current Trends in Natural Sciences (CD-Rom)

ISSN: 2284-9521

ISSN-L: 2284-9521

ISSN-L: 2284-9521

flowering in 25 species of plants, fecundation and even fructification in two species of plants, extension of flowering in seven species of plants (Fig.1-7).

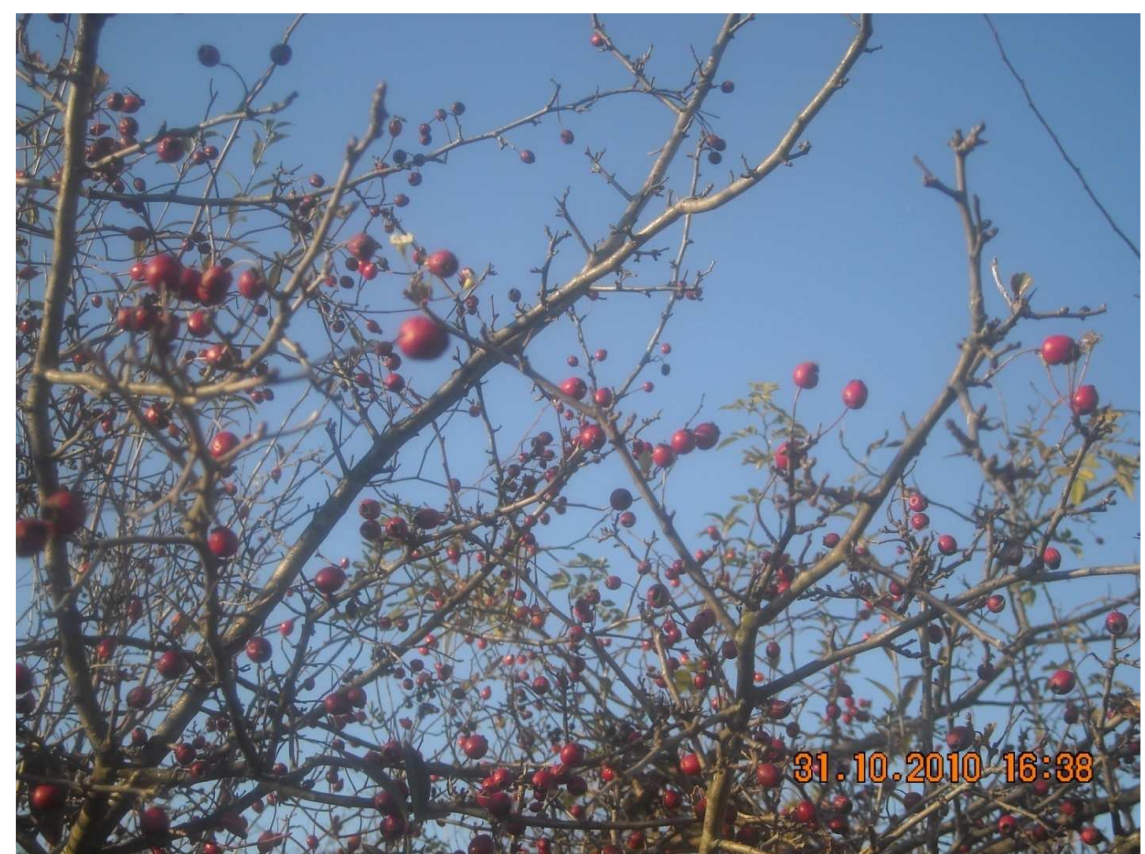

Figure 1. Crataegus monogyna L. (photo Ilie A.L.)

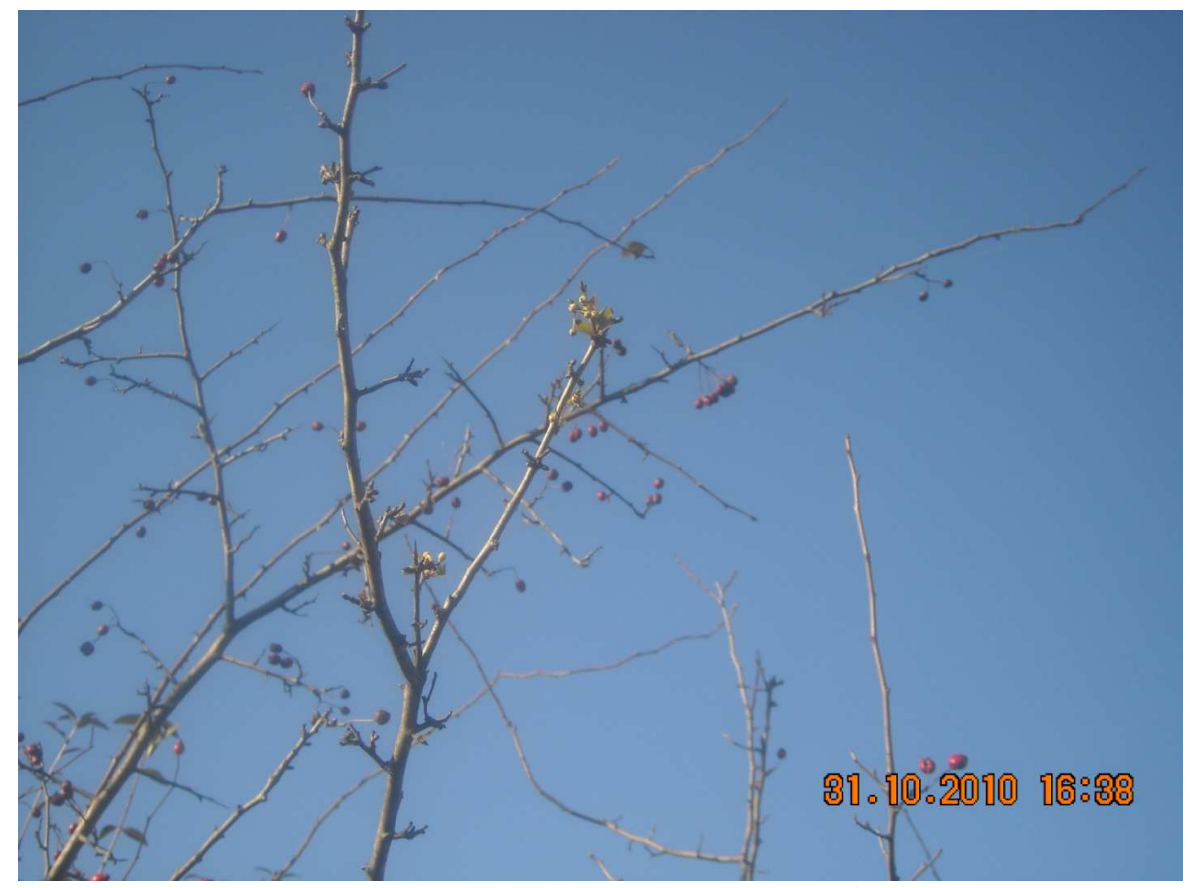

Figure 2. Crataegus monogyna L. (photo Ilie A.L.) 


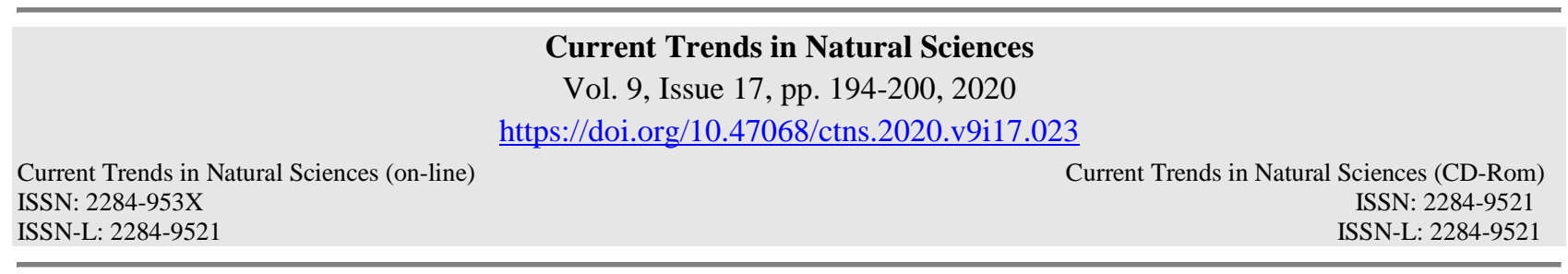

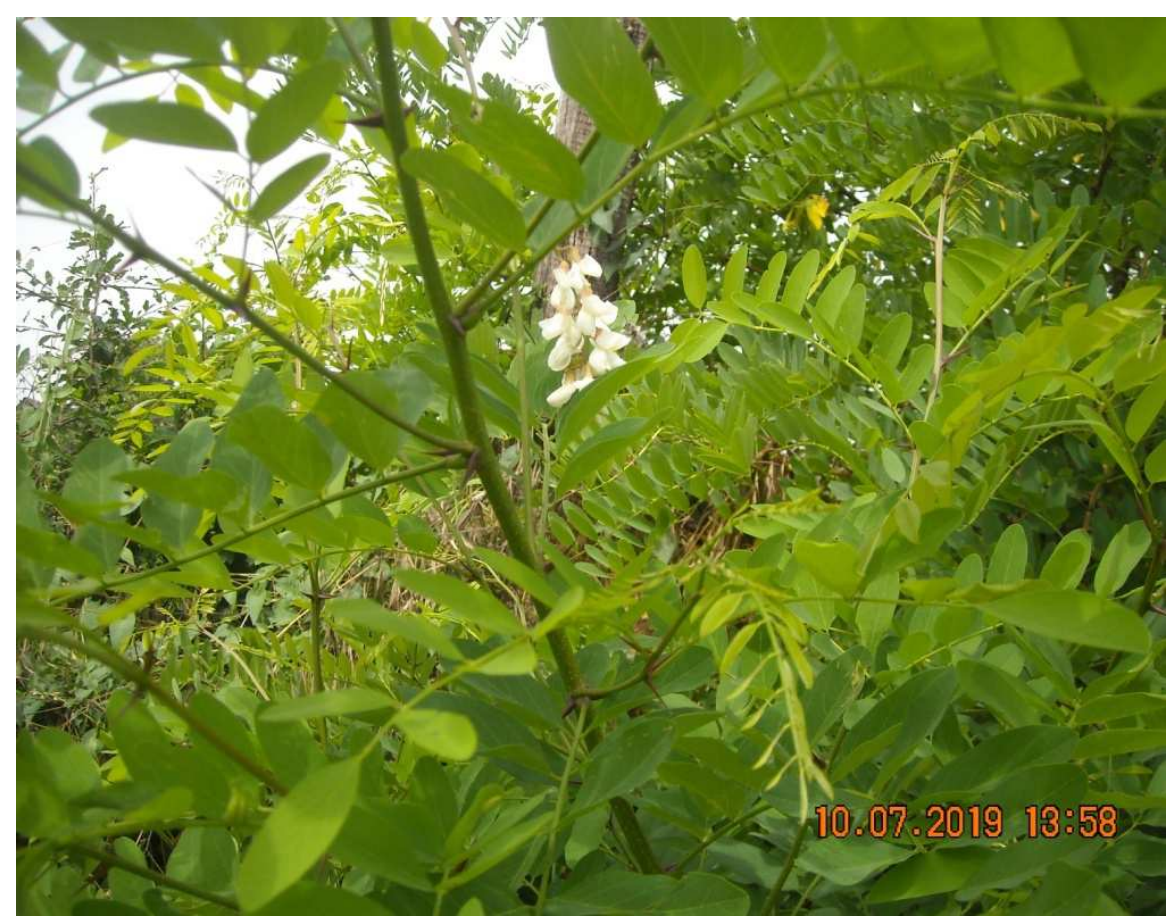

Figure 3. Robinia pseudoacacia L. ( photo Ilie A.L.)

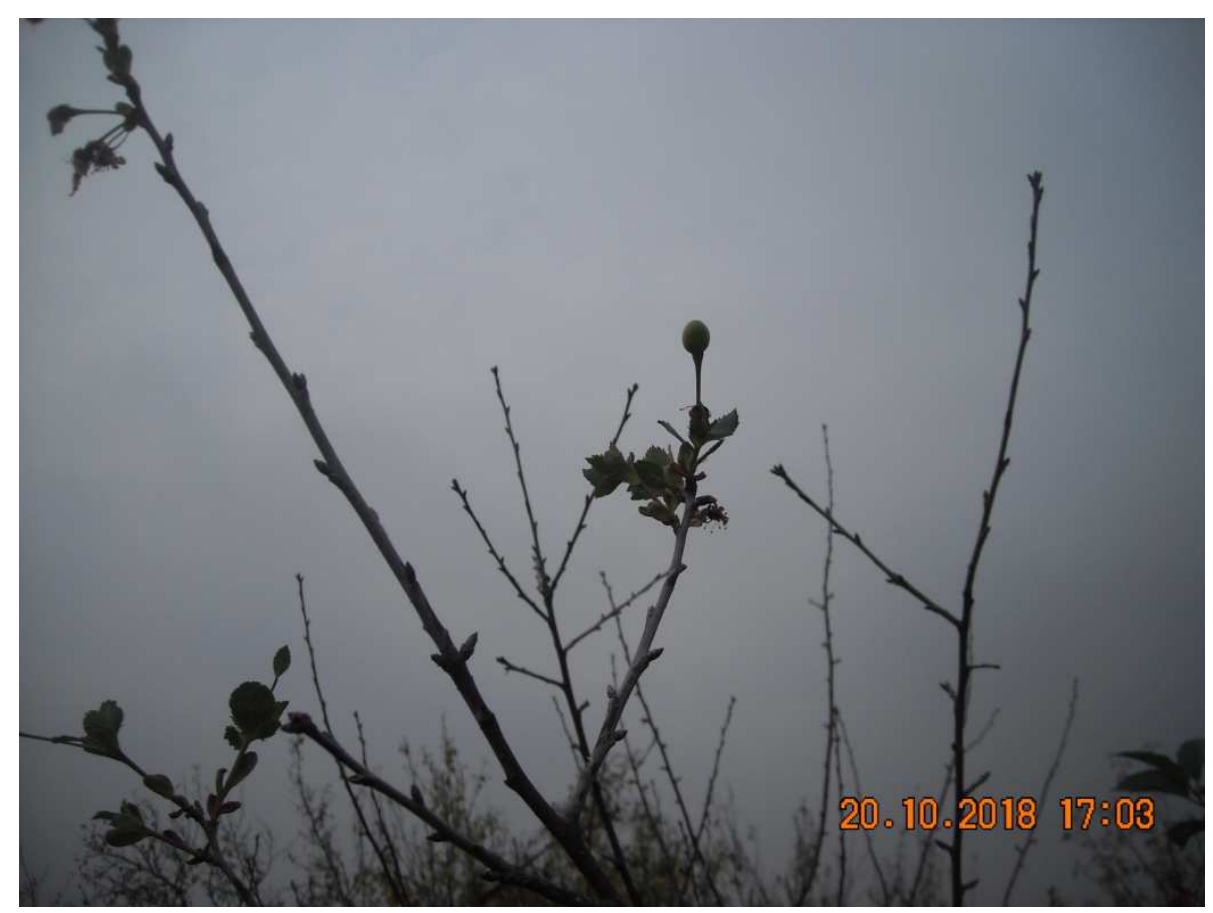

Figure 4. Cerasus avium Moench. - fructification (photo Ilie A.L.) 


\section{Current Trends in Natural Sciences}

Vol. 9, Issue 17, pp. 194-200, 2020

https://doi.org/10.47068/ctns.2020.v9i17.023

Current Trends in Natural Sciences (on-line)

ISSN: 2284-953X

Current Trends in Natural Sciences (CD-Rom) ISSN-L: 2284-9521

ISSN: 2284-9521 ISSN-L: 2284-9521

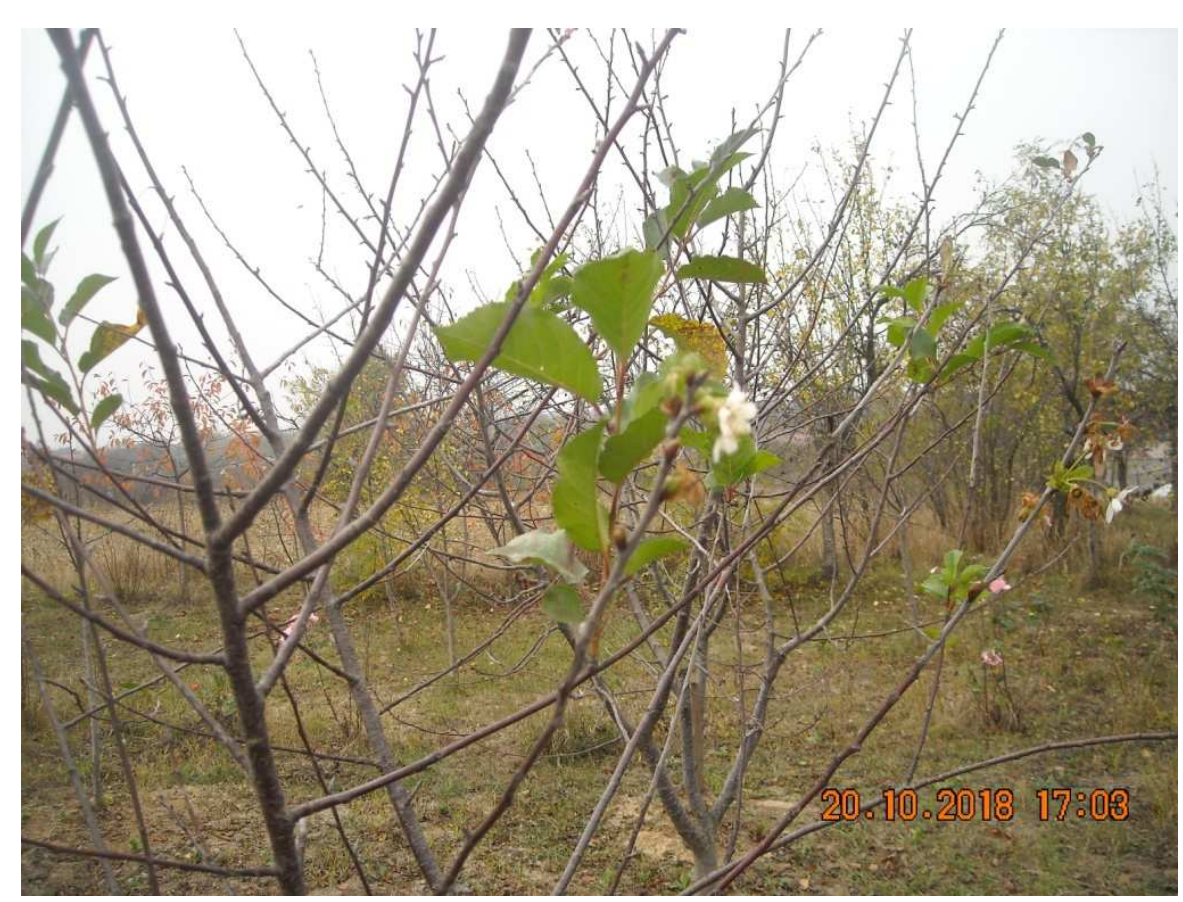

Figure 5. Cerasus avium Moench. - flowering (photo Ilie A.L.)

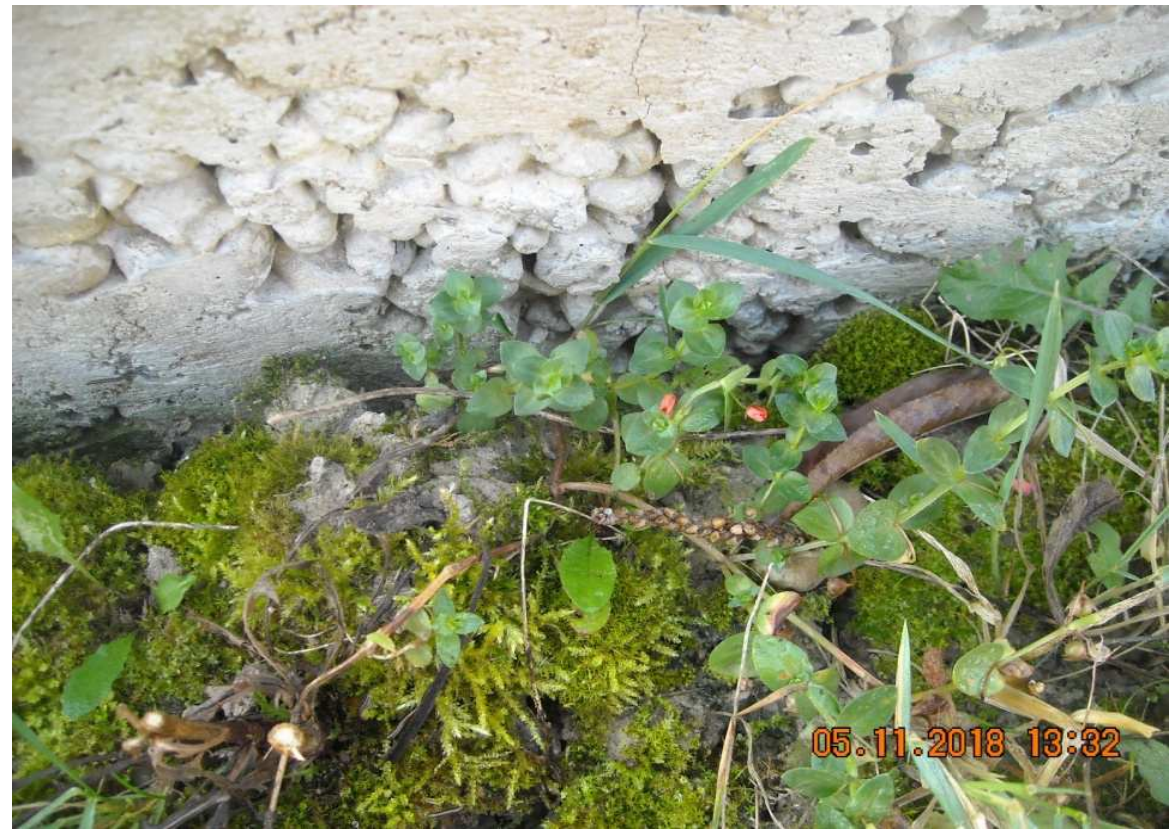

Figure 6. Anagallis arvensis L. (photo Ilie A.L.) 


\section{Current Trends in Natural Sciences}

Vol. 9, Issue 17, pp. 194-200, 2020

https://doi.org/10.47068/ctns.2020.v9i17.023

Current Trends in Natural Sciences (on-line)

ISSN: 2284-953X

Current Trends in Natural Sciences (CD-Rom)

ISSN: 2284-9521

ISSN-L: 2284-9521 ISSN-L: 2284-9521

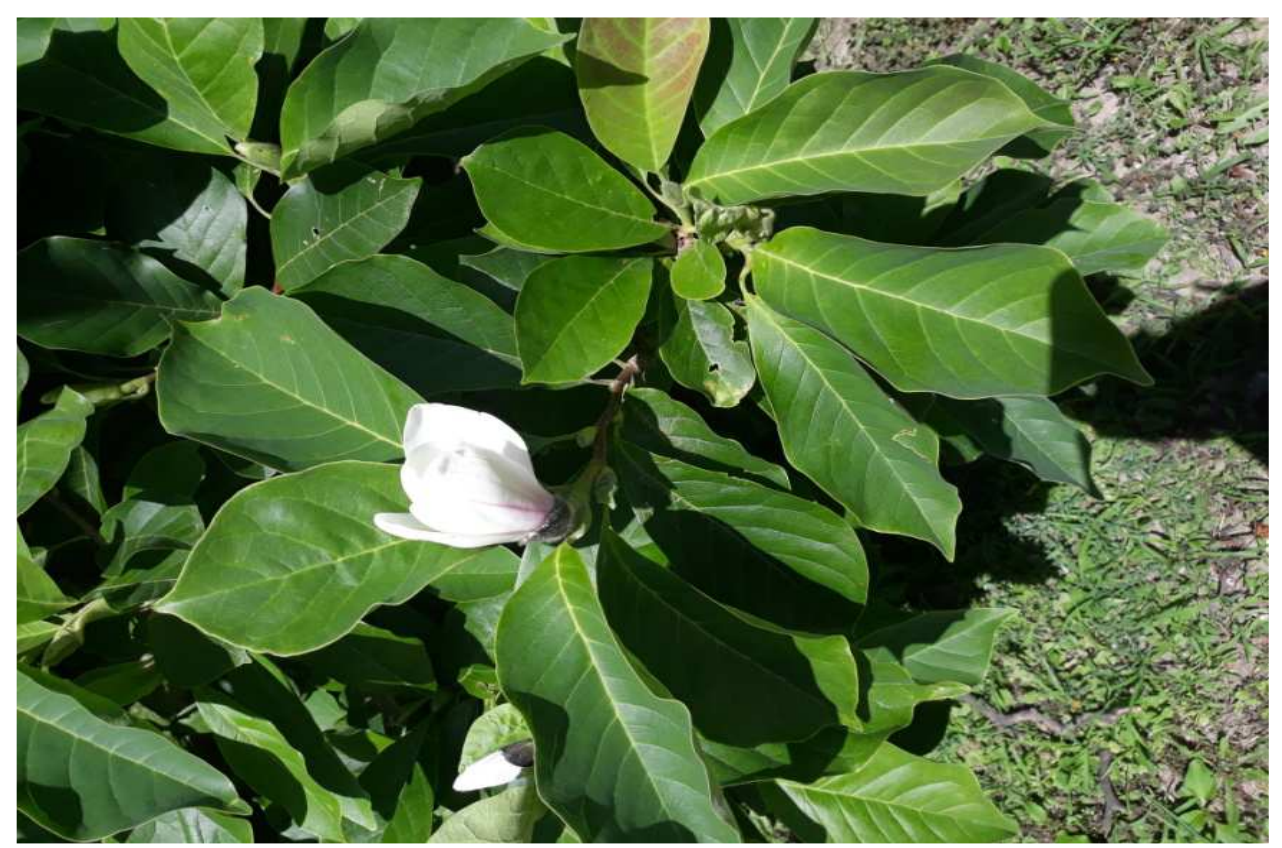

Figure 7. Magnolia denudata Des. (photo Marinescu M.)

\section{CONCLUSIONS}

During 2010-2019, phenological anomalies were identified regarding the flowering of spontaneous and cultivated plants in 35 species from different parts of Romania. The positive temperatures from the cold season and the high temperatures from spring have determined both the premature flowering and flowering extension, sometimes even supplementary flowerings in the cold season (November - December).

\section{REFERENCES}

Ciocârlan, V. (2000). Flora ilustrată a României [The illustrated flora of Romania], Ed. Ceres, Bucureşti.

Ilie, A.L., Năstase, A., Cioboiu O. (2018). Phenological anomalies reagarding the flowering of spontaneous and subspontaneous plants from four different parts of Romania, Muzeul Olteniei, Craiova, Oltenia, Studii şi comunicări, Ştiințele Naturii, 34, 1, 193-199, Craiova.

Menzel A. (2003). Plant phenological anomalies in Germany and their relation to air temperature and NAO,Climatic Change ,57, 243-263.

Wang H., Dai J., Zheng J., Ge Q. (2015). Temperature sensitivity of plant phenology in temperate and subtropical regions of China from 1850 to 2009, International Journal of Climatology, 35, 913-922.

Sârbu, I., Ştefan, N., Oprea, A. (2013). Plante vasculare din România. Determinator ilustrat de teren [Vascular plants from Romania.Illustrated guide], Ed. Victor B.Victor, Bucureşti.

Săvulescu, T. (1952-1976). Flora R.P.R. - Flora R.S.R. [Romanian flora], Ed. Academiei, Bucureşti.

Todor, I. (1968). Mic atlas de plante din flora Republicii Socialiste România [Little guide of plants from Romania], Ed. Didactică şi Pedagogică, Bucureşti. 